\title{
Lung Cancer Screening Uptake: Analysis of BRFSS 2018
}

J Gen Intern Med 36(9):2897-9

DOI: $10.1007 / \mathrm{s} 11606-020-06236-9$

(C) Society of General Internal Medicine 2020

\section{INTRODUCTION}

In 2013, the United States Preventive Services Task Force (USPSTF) recommended annual low-dose CT (LDCT) screening for high-risk smokers. This recommendation was supported by the National Lung Screening Trial that demonstrated reduced lung cancer mortality with screening. ${ }^{1}$

Despite this recommendation, few national surveys have included lung cancer screening in their core measures. The National Health Interview Survey (NHIS), conducted every 5 years, found LDCT rates at 3.9\% in 2015, compared with $3.3 \%$ in 2010 , which predated the recommendation. ${ }^{2}$ The 2017 Behavioral Risk Factor Surveillance Survey (BRFSS) optional lung cancer screening module was distributed in only 11 states, and demonstrated that screening increased to $14.4 \% .^{3}$ At present, there is scant information regarding racial disparities in lung cancer screening. However, historically, there have been racial disparities in other cancer screenings and outcomes. In light of these disparities and limited LDCT screening utilization, examining the racial distribution and characteristics associated with uptake of lung cancer screening is particularly important.

\section{METHODS}

BRFSS is a telephone health survey of US residents that provides state-level data related to major health conditions. We analyzed 2018 BRFSS data and measured self-reported annual LDCT imaging for lung cancer among individuals eligible according to USPSTF criteria (asymptomatic individuals aged 55 to 80 with a 30 pack-year smoking history who currently smoke or quit within the last 15 years) and other screening services. The lung cancer screening questions were an optional survey module included by eight states and individuals with missing eligibility data were excluded. Data was weighted to match state population estimates. All analysis was conducted with SAS version 9.4 (SAS Institute Inc., Cary, $\mathrm{NC)}$ and Rao-Scott $\chi^{2}$ tests were used for comparisons, with

Received June 12, 2020

Accepted September 10, 2020

Published online September 21, 2020 findings considered significant at $p<0.05$. This study was an analysis of a publicly available, deidentified dataset.

\section{RESULTS}

In 2018 BRFSS, 224,679 or $17.7 \%$ of $1,273,013$ USPSTF criteria-eligible smokers reported annual LDCT screening. We found no significant differences in sex, marital status, race, education, or income between the eligible and screened groups $(p>0.05$ for all). We found a higher proportion of respondents with insurance, $\mathrm{COPD}$, and having a $\mathrm{PCP}$ in the screened group. The LDCT-eligible cohort (64.83\%) had lower rates of colon cancer screening compared with non-eligible (73.90\%) ( $p=0.002)$, while breast cancer screening and pneumococcal vaccination were not significantly different (Table 1).

Across states, we found Maine had the lowest $(8.5 \%)$ and Texas the highest $(24.3 \%)$ screening rates among eligible individuals $(p=0.01)$. There were too few non-White respondents to accurately compare the screening prevalence between White and Black, Asian, American/Alaskan, Indian, Hispanic, or other individuals in seven out of eight states. No disparities were found in screening rates between White and Black individuals in Maryland.

\section{DISCUSSION}

Lung cancer screening adoption is slowly increasing, but there remains insufficient information to understand whether screening is reaching those who stand to benefit the most. There are known regional variations in care and disparities wherein minorities receive less treatment with worse lung cancer outcomes, ${ }^{4}$ but limited comparable data by race prevented an accurate assessment of differences. More respondents in the screened group had a PCP and health insurance, corresponding with healthcare access, which is consistent with past BRFSS studies where uninsured individuals were less likely to receive lung ${ }^{3}$ and other cancer (breast, cervical, colorectal) screenings. ${ }^{5}$ This may be an important barrier to further lung cancer screening uptake as the percentage of uninsured individuals in the USA rose in 2018, reversing the downward trend of uninsured rates following the implementation of the Affordable Care Act. The lower rates of colorectal cancer screening among the LDCT-eligible cohort suggest additional education and targeted strategies may be required to increase lung cancer screening rates in this population, 
Table 1 Characteristics of USPSTF Criteria-Eligible Smokers for LDCT Screening

\begin{tabular}{|c|c|c|c|}
\hline Characteristic & Total eligible & Received screening & $\overline{p \text { value }}$ \\
\hline LDCT screening eligible & $n=3007 ;$ weighted $n=1,273,013$ & $n=405 ;$ weighted $n=224,679$ & \\
\hline Total $(\%, 95 \% \mathrm{CI})$ & & $17.65(13.05-22.25)$ & \\
\hline Age (median, IQR) & $63.68(10.44)$ & $62.57(10.07)$ & \\
\hline Male $(\%, 95 \% \mathrm{CI})$ & $49.22(44.10-54.35)$ & $44.09(29.19-58.99)$ & 0.17 \\
\hline Married $(\%, 95 \% \mathrm{CI})$ & $50.86(45.68-56.05)$ & $46.37(31.90-60.84)$ & 0.48 \\
\hline \multicolumn{4}{|l|}{ Race $(\%, 95 \%$ CI $)$} \\
\hline White & $85.96(81.45-90.46)$ & $84.12(71.09-97.14)$ & 0.42 \\
\hline Black & $5.42(3.85-6.98)$ & $5.16(1.48-8.83)$ & \\
\hline Asian & $*$ & $*$ & \\
\hline American Indian/Alaskan native & $1.83(0.82-2.83)$ & $1.09(0.00-2.18)$ & \\
\hline Hispanic & & & \\
\hline Other & $1.59(0.32-2.86)$ & $0.29(0.04-0.53)$ & \\
\hline Education: attend college and higher $(\%, 95 \% \mathrm{CI})$ & $46.63(41.88-51.39)$ & $42.39(27.67-57.11)$ & 0.68 \\
\hline Income $>15,000(\%, 95 \% \mathrm{CI})$ & $74.02(69.60-78.45)$ & $79.20(66.58-91.82)$ & 0.69 \\
\hline Pack years (median, IQR) & $45.80(22.26)$ & $48.28(29.80)$ & \\
\hline Current smoker $(\%, 95 \% \mathrm{CI})$ & $55.77(51.01-60.53)$ & $58.98(44.58-73.38)$ & 0.63 \\
\hline Health plan coverage $(\%, 95 \% \mathrm{CI})$ & $91.89(89.39-94.40)$ & $97.01(94.50-99.52)$ & 0.008 \\
\hline COPD $(\%, 95 \% \mathrm{CI})$ & $35.36(30.18-40.54)$ & $52.63(37.49-67.78)$ & 0.007 \\
\hline PCP $(\%, 95 \% \mathrm{CI})$ & $88.64(85.30-91.99)$ & $97.65(95.12-100.00)$ & $<0.001$ \\
\hline \multicolumn{4}{|l|}{ Other preventative health } \\
\hline \multicolumn{4}{|l|}{ Mammography $(\%, 95 \%$ CI $)$} \\
\hline \multirow{2}{*}{\multicolumn{2}{|c|}{$\begin{array}{l}\text { LDCT screening eligible } \\
n=1502 ; \text { weighted } n=650,103\end{array}$}} & $64.71(57.33-72.09)$ & 0.14 \\
\hline & & & \\
\hline & & $70.29(68.09-72.50)$ & \\
\hline$n=18,351 ;$ weighted $n=6,953,069$ & & & \\
\hline \multicolumn{4}{|l|}{ Colon cancer screening $(\%, 95 \% \mathrm{CI})$} \\
\hline \multirow{2}{*}{\multicolumn{2}{|c|}{$n=2515 ;$ weighted $n=1,106,218$}} & $64.83(59.17-70.49)$ & 0.002 \\
\hline & & & \\
\hline \multicolumn{2}{|l|}{$\begin{array}{l}\text { Non-LDCT screening eligible } \\
n=21,355 ; \text { weighted } n=8,957,348\end{array}$} & $73.90(71.81-75.98)$ & \\
\hline \multicolumn{4}{|l|}{$\begin{array}{l}n=21,355 ; \text { weighted } n=8,957,348 \\
\text { Pneumococcal vaccination for }>65(\%, 95 \% \mathrm{CI})\end{array}$} \\
\hline \multirow{2}{*}{\multicolumn{2}{|c|}{$\begin{array}{l}\text { LDCT screening eligible } \\
n=1758 ; \text { weighted } n=625,235\end{array}$}} & $68.76(61.82-75.70)$ & 0.34 \\
\hline & & & \\
\hline \multicolumn{2}{|l|}{ Non-LDCT screening eligible } & $65.15(62.87-67.42)$ & \\
\hline
\end{tabular}

Data presented as weighted median (IQR) or weighted \% (95\% confidence intervals); *too small denominator to be accurately estimated based on BRFSS guidelines: $<50$ unweighted respondents

particularly because of stigma and distrust among long-term smokers. ${ }^{6}$

This study provides an update on LDCT screening uptake and is the first to look at the differences by race/ethnicity by state. BRFSS data was self-reported, which brings in possible recall bias and is limited to those accessible by telephone. Only eight states had the optional lung cancer screening module, which limits this study's generalizability, and there were limited non-White participants, suggesting that placing lung cancer screening within the core questions of all state and national datasets is important to understand adoption and implementation.

Dustin Kee, $B A^{1}$

Juan Wisnivesky, $M D, D r P H^{2}$

Minal S. Kale, MD, $M P H^{2}$

${ }^{1}$ Icahn School of Medicine at Mount Sinai, ,

New York, NY, USA

${ }^{2}$ Division of General Internal Medicine, Icahn School of Medicine at Mount Sinai,

New York, NY, USA
Corresponding Author: Dustin Kee, BA; Icahn School of Medicine at Mount Sinai, New York, NY, USA (e-mail: dustin.kee@icahn.mssm.edu).

Funding The work was supported by a grant from the National Institutes of Health: NIH- KO7 CA187071 and an American Cancer Society Research Scholar Grant.

Data Availability The datasets analyzed during the current study are publicly available at the CDC's website, under the 2018 BRFSS optional modules, https://www.cdc.gov/brfss/annual_data/2018/ llcp_multiq.html.

\section{Compliance with Ethical Standards:}

Conflict of Interest: Mr. Kee has nothing to disclose. Dr. Kale reports grants from the American Cancer Society, outside the submitted work. Dr. Wisnivesky reports personal fees from Sanofi, personal fees from GlaxoSmithKline, and personal fees from Banook, and grants from Sanofi, outside the submitted work.

\section{REFERENCES}

1. National Lung Screening Trial Research T, Aberle DR, Adams AM, et al. Reduced lung-cancer mortality with low-dose computed tomographic screening. N Engl J Med 2011;365(5):395-409.

2. Jemal A, Fedewa SA. Lung Cancer Screening With Low-Dose Computed Tomography in the United States-2010 to 2015. JAMA Oncol 2017;3(9):1278-1281. 
3. Zahnd WE, Eberth JM. Lung Cancer Screening Utilization: A Behavioral Risk Factor Surveillance System Analysis. Am J Prev Med 2019;57(2):250255.

4. Wolf A, Alpert N, Tran BV, Liu B, Flores R, Taioli E. Persistence of racial disparities in early-stage lung cancer treatment. J Thorac Cardiovasc Surg. 2019;157(4):1670-1679.e1674.

5. Zhao G, Okoro CA, Li J, Town M. Health Insurance Status and Clinical Cancer Screenings Among U.S. Adults. Am J Prev Med 2018;54(1):e11e19.
6. Carter-Harris L, Ceppa DP, Hanna N, Rawl SM. Lung cancer screening: what do long-term smokers know and believe? Health Expect 2017;20(1):59-68.

Publisher's Note: Springer Nature remains neutral with regard to jurisdictional claims in published maps and institutional affiliations. 